\title{
Protection and Dissection of Recurrent Laryngeal Nerve in Salvage Thyroid Cancer Surgery to Patients with Insufficient Primary Operation Extent and Suspicious Residual Tumor
}

\author{
Wen-Bin Yu*, Nai-Song Zhang
}

\begin{abstract}
Some thyroid cancer patients undergone insufficient tumor removal in the primary surgery in China .our aim is to evaluate the impact of dissection of the recurrent laryngeal nerve during a salvage thyroid cancer operation in these patients to prevent nerve injury. Clinical data of 49 enrolled patients who received a salvage thyroid operation were retrospectively reviewed. Primary pathology was thyroid papillary cancer. The initial procedure performed included nodulectomy ( 20 patients), partial thyroidectomy (19 patients) and subtotal thyroidectomy (10 patients). The effect of dissection and protection of the recurrent laryngeal nerve and the mechanism of nerve injury were studied. The cervical courses of the recurrent laryngeal nerves were successfully dissected in all cases. Nerves were adherent to or involved by scars in 22 cases. Three were ligated near the place where the nerve entered the larynx, while another three were cut near the intersection of inferior thyroid artery with the recurrent laryngeal nerve. Light hoarseness occurred to four patients without a preoperative voice change. In conclusion, accurate primary diagnosis allows for a sufficient primary operation to be performed, avoiding insufficient tumor removal that requires a secondary surgery. The most important cause of nerve damage resulted from not identifying the recurrent laryngeal nerve during first surgery, and meticulous dissection during salvage surgery was the most efficient method to avoid nerve damage.
\end{abstract}

Keywords: Thyroid cancer - surgery - thyroid papillary cancer

Asian Pac J Cancer Prev, 16 (17), 7457-7461

\section{Introduction}

Ipsilateral lobectomy with isthmectomy and level VI dissection is the basic operation for patients with thyroid papillary cancer confined to one lobe(1). Identification of the recurrent laryngeal nerve prior to lobectomy and level VI dissection is crucial for nerve protection. However, many cases were treated as benign tumor without preoperative or perioperative confirmation of thyroid papillary cancer resulting incomplete tumor removal, which may require a salvage operation. Herein, we described our surgical experience with dissection and protection of the recurrent laryngeal nerve by retrospectively reviewing 49 cases with suspicion of residual tumor.

\section{Materials and Methods}

\section{Patients}

Forty-nine patients who underwent secondary surgery to remove the residual tumor from January 2007 through December 2008 were enrolled in this study. Suspicion of tumor in the residual thyroid lobe or lymph nodes in the level VI region was determined by clinical examination and/or imaging findings (CT and high-resolution neck ultrasound examination). All of the patients were initially treated with a primary operation to remove a benign tumor at other medical institutes. The diagnosis of thyroid papillary cancer was confirmed by pathological examination at our institute for all patients. There were 9 men and 40 women with age ranging from 17 to 66 years and a median age of 34.5 years. Patients were initially treated with nodulectomy in 20 , partial thyroidectomy in 19 , and subtotal thyroidectomy in 10 cases. Identification of the recurrent laryngeal nerve, any kind of neck dissection and perioperative frozensection pathological examination were not found in the initial operative records. Hoarseness occurred in 6 cases postoperatively and vocal cord paralysis was confirmed by fiberoptic laryngoscopy.

\section{Surgical planning}

All patients referred to our institution received a CT and high-resolution neck ultrasound examination, and residual tumor in the remainder thyroid gland and level $\mathrm{VI}$ region was suspected in the patients included in this 
study. Our basic salvage operation consists of an ipsilateral lobectomy with isthmectomy and level VI dissection. Routine exploration of the ipsilateral neck was carried out for suspected lymph node metastasis and tissue sent for intra-operative pathology examination. Ipsilateral radical neck dissection or modified radical neck dissection was performed in patients with positive lymph node metastasis; no elective lateral neck dissection was performed.

\section{Identification of the recurrent laryngeal nerve}

The infrahyoid strap musculature was resected according to suspected tumor contamination at the level of cricoid cartilage superiorly and sternal notch inferiorly. The carotid artery was dissected from the level of thyroid cartilage down to clavicle. Then the level VI region was fully exposed. The recurrent laryngeal nerves were most frequently identified in areas previously lacking or with minimal dissection. The nerve was dissected in the tracheoesophageal groove from the level of the sternal notch, which was not touched in the first operation (Figure 1). The identity of the nerve was confirmed by the white color and longitudinal course within the tracheoesophageal groove. Meticulous microdissection of the nerve was carried out from the clavicle up into the laryngeal inlet, to identify the frequently arborized laryngeal branches and release them in their entirety. No ligations were made before nerve confirmation and release was done where previous ligation of nerve existed. Ligation of vessels and other tissues was made by 3-0 sutures to avoid or decrease scar proliferation, which could affect recurrent laryngeal nerve function. No coagulation was performed near the nerve to avoid electrical conduction resulting in nerve damage. When parathyroid tissue was identified distinct from recurrent carcinoma, it was immediately finely minced and sent for frozen section pathology review. Parathyroid glands confirmed by pathology would be considered for autotransplantation into the sternocleidomastoid muscle. The lymph-fatty tissue of level VI and ipsilateral residual lobe were dissected en bloc in the tracheoesophageal groove after retraction and protection of the nerve by euthyphoria (Figure 2)

\section{Statistical analysis}

SPSS 11.5 software was used for all statistical analysis. $\mathrm{P}<0.05$ was considered to be statistical significant. Fisher's exact test was used to explore the relationship of hoarseness after the first operation, the number of nerves involved in scar tissue, and hoarseness after the salvage operation among the three primary surgical approaches: nodulectomy, partial thyroidectomy and subtotal thyroidectomy.

\section{Results}

The ipsilateral cervical course of the recurrent laryngeal nerve was successfully dissected in all 49 cases. For the 20 patients with a primary nodulectomy, nerves were found to be unaffected by the prior operation in 16 patients, and no damage occurred after the salvage operation. Nerves were adherent to prior scar in 4 cases, and slight hoarseness occurred with one patient because the region of adherence was located near the nerve inlet. Of the 19 patients with an initial partial thyroidectomy, no nerve damage occurred for the 11 patients who underwent an anterior and internal thyroid partial resection. The other 8 patients underwent inferior and superior thyroid partial resection and the nerve was tightly adherent to the scar. The nerve was ligated in two cases, one nerve ligation near nerve inlet (Figure 3)and one cut at the level of inferior thyroid artery ,One of the eight patients without preoperative nerve damage developed slight hoarseness after the salvage operation. Severe scar was

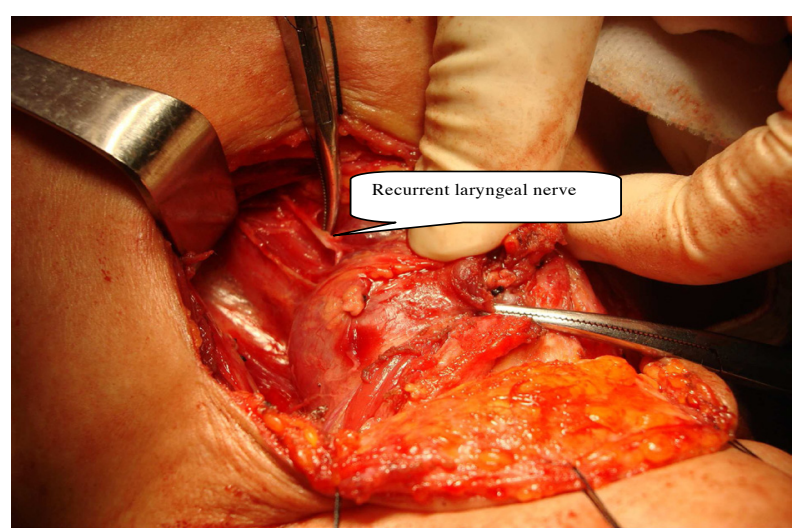

Figure 1. Dissection of the Recurrent Laryngeal Nerve in the Tracheoesophageal Groove from the Level of Sternal Notch, a Region not Explored in the First Operation. (in black \& white)

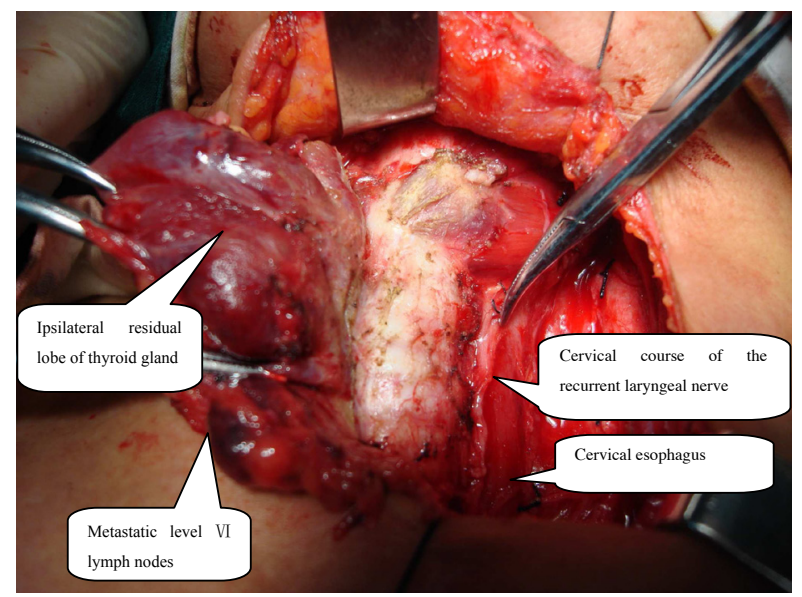

Figure 2. En Bloc Resection of Lymph and Fatty Tissue of Level VI and Ipsilateral Residual Thyroid Gland Lobe. (in black \& white)

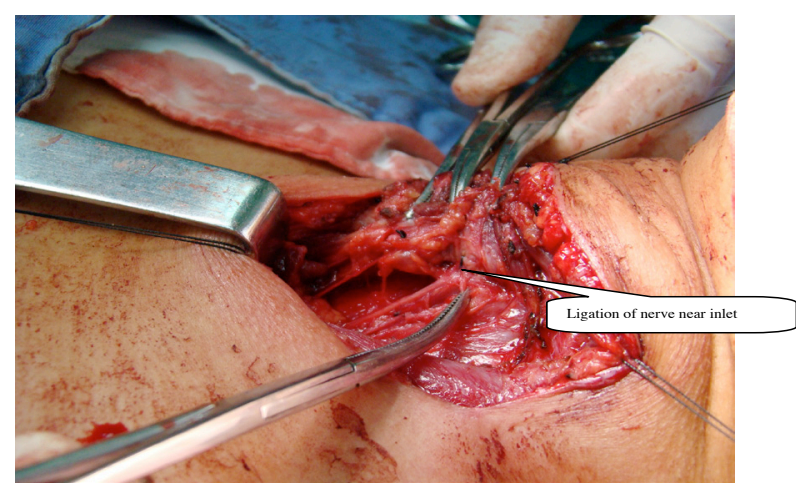

Figure 3. Recurrent Laryngeal Nerve Ligation Near Nerve Inlet. (in black \& white) 
Table 1. Recurrent Laryngeal Nerve Injury with Three Types of Primary Surgery

\begin{tabular}{lcccc}
\hline Type of primary surgery & $\begin{array}{c}\text { Nodulectomy } \\
\%(\mathrm{~N})\end{array}$ & $\begin{array}{c}\text { Partial thyroidectomy } \\
\%(\mathrm{~N})\end{array}$ & $\begin{array}{c}\text { Subtotal thyroidectomy } \\
\%(\mathrm{~N})\end{array}$ & $\mathrm{p}$ value \\
\hline Hoarseness after initial operation & $0 \%(0)$ & $10.5 \%(2)$ & $40 \%(4)$ & 0.005 \\
Nerves involved in scar tissue & $20 \%(4)$ & $42.1 \%(8)$ & $100 \%(10)$ & 0.005 \\
Hoarseness after salvage operation & $5 \%(1)$ & $5.89 \%(1)$ & $33.3 \%(2)$ & 0.179 \\
\hline
\end{tabular}

found in the previously operated thyroid bed for the 10 patients with subtotal thyroidectomy. These nerves were tightly adherent to or involved in the scar, which led to a difficult dissection of the recurrent laryngeal nerves. In the four patients with hoarseness (and nerve damage confirmed by fiberoptic laryngoscopy) after first operation, two were found to have cut nerves at the level of inferior thyroid artery and the other two had nerves sutured and ligated near the nerve inlet. The two ligated nerves were released and these patients had greatly improved voice postoperatively. Two patient without preoperative nerve damage developed slight hoarseness after the salvage operation.

Table 1 shows nerve injury after nodulectomy, partial thyroidectomy and subtotal thyroidectomy. Significant statistical differences among these three groups were observed for the development of hoarseness after first operation and scars involving the nerves, while the risk of developing hoarseness after salvage operation was not affected by the initial operation.

\section{Discussion}

Ipsilateral lobectomy with isthmectomy and levelVI dissection is the basic operation for patients with thyroid papillary cancer located in one lobe[1]. Recurrent laryngeal nerve identification during the operation is a necessary and key step for lobectomy and levelVI dissection by the en bloc principle(2-4). A malignant diagnosis of a thyroid mass, familiarity with the anatomy, and skill at identifying the recurrent laryngeal nerve are the key to nerve dissection.

In China, many thyroid cancer patients are misdiagnosed with a benign tumor preoperatively and perioperatively and then treated without routine identification of the recurrent laryngeal nerve. Preoperative and perioperative confirmation of thyroid papillary cancer is crucial to determine the appropriate surgical modality. If the diagnosis is made postoperatively, then the question is only whether residual tumor exists and if a salvage operation is now necessary. Preoperative diagnosis consists mainly of clinical examinations, imaging including CT and high-resolution neck ultrasound, and fine-needle aspiration biopsy with pathology. Clinical characteristics consistent with malignancy include: hard or fixed mass, irregular surface and unclear margins. Ultrasonography is very useful for detecting malignant thyroid cancer (5-7). Typical ultrasonographic features of malignancy include: diameter $1 \mathrm{~cm}$ or more, clear hypoechoic or nonhomogeneous pattern with alternating hypoechoic and hyperechoic areas, irregular cystic appearance, presence of internal microcalcification, and rounded or bulging shape with increased an anteroposterior diameter. CT is a useful adjuvant tool when suspicion for malignancy is high. Malignant CT characteristics include: irregular shape, untidy margins, peninsular tubercles around the tumor, absence of a complete enhancing ring around the tumor, fine globular calcifications and infiltration of adjacent structures(8-12). Fine-needle aspiration biopsy is the most effective modality to diagnose thyroid cancer (13-17) with a reported sensitivity of $92.3 \%$. It is widely used for masses greater than $1 \mathrm{~cm}$ in diameter. During the operation, tumors found to be unencapsulated, solid, hard, and grey-white or grey-red features should be suspected to be malignancy. Having a preoperatively suspicious mass in combination with perioperative gross inspection and perioperative frozen-section pathological examination consistent with malignancy is sufficient to confirm most malignant thyroid masses. If this strategy were applied for patients treated for benign thyroid tumors, fewer salvage operations would be performed. All forty-nine cases in our study were misdiagnosed as a benign tumor preoperatively without perioperative gross inspection or frozen-section pathological examination.

Thyroid nodules are very common in the general population(18-21). The reported incidence rate of thyroid nodules is about $4 \sim 7 \%$ by palpation and $50 \sim 70 \%$ by high-resolution neck ultrasound. Among those, malignant nodules account for only $4 \sim 7 \%$. The high incidence of benign nodules and low incidence of malignant nodules results in poor recognition and treatment of thyroid cancer by many surgeons in China. Many surgeons operate without identification of the recurrent laryngeal nerve, which leads to scar proliferation and makes nerve dissection very difficult in salvage operations. Surgeons leave some thyroid tissue on thyroid posterior capsule to prevent nerve damage. However, the manipulation required to control bleeding of the residual gland on the posterior capsule has a risk of causing damage to the unidentified recurrent laryngeal nerve, while also possibly leaving residual cancer behind.

Salvage thyroid surgery is more technically demanding because of the presence of scar tissue and distorted anatomy(22), which may result in a greater risk of injury to the recurrent laryngeal nerve. When the initial surgery was distant from the nerve, the effect on the nerve was relatively small and nerve dissection was easier. If the first surgery involved or damaged the nerve, injury to the recurrent laryngeal nerve may still occur during the salvage operation and may be severe, despite full dissection of the cervical course of the nerve. Based on our experience, we consider the cervical recurrent laryngeal nerve as having two regions that are divided by the inferior thyroid artery. The part above the artery is tightly associated with the thyroid gland, and the nerve is more likely to be affected or injured in this region by first 
operation. The part below inferior thyroid artery would not be explored by the initial thyroid operation, so we choose this region to begin our dissection in the salvage operation. The nerve was dissected in tracheoesophageal groove below the inferior thyroid artery and its identity confirmed by the white color and longitudinal course in the groove. The inferior thyroid artery in its course to the thyroid gland, after crossing the recurrent laryngeal nerve, has several branches that anastomose with each other and form a fine mesh-like structure $(23,24)$. When manipulation occurs in this region, there is a high risk of bleeding and an unidentified recurrent laryngeal nerve could easily be injured when achieving hemostasis. In our study, three nerves were found cut at the level of inferior thyroid artery, which likely was caused by suturing or clamping for hemostasis without identification of the recurrent laryngeal nerve. The dissection of the nerve was very difficult during the salvage operation because there was adhesion with the scar generated from the first operation. A more extensive first operation resulted in more severe adhesion with the nerve, until in some cases it was completely encapsulated by scar. In our study, there were significant differences among the three groups for hoarseness after the first operation, though not after the salvage operation. The number of nerves affected by scars also differed depending on the primary surgery. One-third of patients developed hoarseness in the subtotal thyroidectomy group, a higher proportion than the two other surgical groups. When the nerve was tightly adherent to or involved by the scar, nerve dissection was performed by scalpel or tissue scissors. Rather than close dissection, in some cases, small amounts of scar tissue were left along the nerve for protection . Despite this, some patients still became hoarse, possibly because dissection within the scar tissue could have led to mechanical injury and decreased blood supply. The risk of injury to the recurrent laryngeal nerve during the salvage operation was increased greatly by the scar tissue generated by the first operation, highlighting the importance of diagnosing thyroid cancer prior to surgery. This way, the first surgical exploration can be of sufficient extent to decrease or avoid the need for and risks of a salvage operation.

Near the laryngeal inlet, the recurrent laryngeal nerve can be easily damaged because the nerve branches have more anatomic variability $(24,25)$, and the venous plexus between the posterior surface of the superior thyroid gland and the inferior constrictor muscle of pharynx is prone to hemorrhage during nerve dissection. In this study, three nerves were found sutured and ligated near the nerve inlet, and suturing the bleeding residual gland together with the nerve is the likely mechanism of these nerve injuries.

In conclusion, some thyroid cancer patients in China received an operation for a benign tumor that did not involve identification of the recurrent laryngeal nerve and required a salvage operation for residual cancer with the associated high risk of nerve injury. An appropriate primary diagnosis allows for a sufficient primary operation and avoids the need for a second salvage operation. Lack of identification of the recurrent laryngeal nerve is the most important cause of nerve damage, and meticulous nerve dissection is still the most efficient method to avoid nerve injury.

\section{Acknowledgements}

The authors would like to thank all study investigators, coordinators and patients who participated in the study.

\section{References}

Ahn JE, Lee JH, Yi JS, et al (2008). Diagnostic accuracy of $\mathrm{CT}$ and ultrasonography for evaluating metastatic cervical lymph nodes in patients with thyroid cancer. World J Surg, 32, 1552-8.

Antonelli A, Miccoli P, Fallahi P, et al (2003). Role of neck ultrasonography in the follow-up of children operated on for thyroid papillary cancer. Thyroid, 13, 479-84.

Antonelli A, Miccoli P, Ferdeghini M, et al (1995). Role of neck ultrasonography in follow-up of patients operated on for different thyroid cancer. Thyroid, 5, 25-9,

Ardito G, Revelli L, D'Alatri L, et al (2004) Revisited anatomy of the recurrent laryngeal nerves. Am J Surg, 187, 249-53.

Ashkan BS, Daniel MD, Sivakumar MD (2002). Mircosurgical anatomy of the laryngeal nerves as related to thyroid surgery. Laryngoscope, 112, 386-92.

Chiang FY, Wang LF, Huang YF, Lee KW, Kuo WR (2005). Recurrent laryngeal nerve palsy after thyroidectomy with routine identification of the recurrent laryngeal nerve. Surgery, 137, 342-7.

Cooper DS, Doherty GM, Haugen BR, et al (2006). Tuttle RM and American ThyroidAssociation Guidelines Taskforce: Management guidelines for patients with thyroid nodules and differentiated thyroid cancer. Thyroid, 16, 109-42.

Davies L and Welch HG (2006). Increasing incidence of thyroid cancer in the United States 1973-2002. JAMA, 295, 2164-7.

Deandrea M, Mormile A, Veglio M, et al (2002). Fine needle aspiration biopsy of thethyroid: comparison between thyroid palpation and ultrasonography. Endocr Pract, 8, 282-6.

Haberal I, Celik H, Gocmen H, et al (2004). Which is important in the evaluation of metastatic lymph nodes in head and neck cancer: palpation, ultrasonography, or computed tomography? Otolaryngol Head Neck Surg, 130, 197-201.

Heller KS (2007). Do all cancers need to be treated? The role of thyroglobulin in the management of thyroid cancer: the 2006 Hayes Martin lecture. Arch Otolaryngol Head Neck Surg, 133, 639-43.

Hermann M, Alk G, Roka R, Glaser K, Freissmuth M (2002). Laryngeal recurrent nerve injury in surgery for benign thyroid disease: effect of the nerve dissection and impact of individual surgeon in more than 27,000 nerves at risk. Ann Surg, 35, 261-8.

Ito Y, Tomoda C, Uruno T, et al (2005). Ultrasound-detectable and anatomopathologically-detectable node metastasis in the lateral compartment as indicators of worse relapse-free survival in patients with papillary thyroid carcinoma. World J Surg, 29, 917-20.

Jeong HS, Baek CH, Son YI, et al (2006). Integrated 18F-FDG $\mathrm{PET} / \mathrm{CT}$ for the initial evaluation of cervical node level of patients with papillary thyroid carcinoma: comparison with ultrasound and contrast-enhanced CT. Clin Endocrinol, 65, 402-7.

Kim MK, Mandel SH, Baloch Z, et al (2004). Morbidity following central compartment reoperation for recurrent or persistent thyroid cancer. Arch Otolaryngol Head Neck Surg, 130, 1214-6.

Kwak JY, Kim EK, Kim HJ, et al (2009). How to combine ultrasound and cytological information in decision making about thyroid nodules. Eur Radiol, 19, 1923-31. 
Layfi eld LJ, Cibas ES, Gharib H, Mandel SJ (2009). Thyroid aspiration cytology: current status. CA Cancer J Clin, 59, 99-110.

Mehanna HM, Jain A, Morton RP, Watkinson J and Shaha A (2009). Investigating the thyroid nodule. BMJ, 338, 705-9.

Moreau S, Goullet de Rugy M, Babin E, et al (1998). The recurrent laryngeal nerve:related vascular anatomy. Laryngoscope, 108, 1351-3.

Seo YL, Yoon DY, Lim KJ, et al (2010). Locally advanced thyroid cancer: can CT help in prediction of extra-thyroidal invasion to adjacent structures? AJR Am J Roentgenol, 195, 240-4.

Uruno T, Miyauchi A, Shimizu K, et al (2005). Usefulness of thyroglobulin measurement in fine-needle aspiration biopsy specimens for diagnosing cervical lymph node metastasis in patients with papillary thyroid cancer. World J Surg, 29, 493-5.

Wai FC, Brian HHL, Chung YL (2006). The role of intraoperative neuromonitoring of recurrent laryngeal nerve during thyroidectomy: A comparative study on 1000 nerves at risk. Surgery, 140, 866-73.

Won YJ, Sung J, Jung KW, et al (2009). Nationwide cancer incidence in Korea 2003-2005. Cancer Res Treat, 41, 122-31.

Xie BK, Guan YB, Yuan XP, Li HG (2003). Relationship between computed tomography (CT) manifestations and pathology in thyroid cancer. Ai Zheng, 22, 192-7.

Yu WB, Zhang NS and Zeng ZY (2009). Use of en bloc principle in re-operation of thyroid carcinoma. Zhong Hua Er Bi Yan Hou Tou Jing Wai Ke Za Zhi, 44, 268-271. 